\title{
The Design of Oil Well Downhole Mechanical Analysis System
}

\author{
Panpan Zhao, Xiufang Wang ${ }^{*}$, Ying Liu, and Mengmeng Wu
}

School of Electrical \& Information Engineering, Northeast Petroleum University, Daqing, 163318, China

\begin{abstract}
With the rapid development of drilling technology, borehole has become increasingly complex demanding higher standards for downhole operation. The downhole mechanical analysis is an important part of the oil well production analysis. It is very important for the process of drilling, completion and work over operations. The previous operation based on experience has been unable to meet the modern production requirement in accuracy. For the present situation of oil field information construction and the problems being confronted in the production process, this paper designs and implements the downhole mechanical analysis system based on .NET. This system can provide quick and accurate analysis results of downhole string in the process of well production and can also provide the basis for equipment selection and scheme selection in the production process.
\end{abstract}

The design of this system mainly includes two parts: part one consists of the analysis of the deformation to the tubular column with the packer and the other part comprises of the analysis of frictional resistance of tubular column. This system has the advantages of simple operation, accurate calculation, clear analytical results and comprehensive analysis.

Keywords: Friction analysis, Mechanics analysis, Packer, Piston effect.

\section{INTRODUCTION}

The downhole mechanics analysis is a very important part of oil well production analysis. There are many researches on the analysis of oil well downhole mechanical processes in China and all over the world. In China, Wang Yong, Liu Shuzhi, Liu Qi, Li Qindao et al. have been researching on the force analysis of packer string [1-3]. In the rest of the world, renowned scholars such as Lubinski, Woods and Hammerlindle have been researching on the analysis of pipe string mechanics [4-6]. Some researchers understand that it reduces the accuracy of oil well production analysis. Therefore, it is very necessary to design a complete oil well downhole mechanical analysis system.

To obtain the downhole mechanical analysis, data is collected and analyzed from two downhole processes. The first process consists of the analysis of the deformation to the tubular column with the packer and the other comprises of the analysis of frictional resistance of tubular column.

\section{THE ANALYSIS OF THE DEFORMATION TO THE TUBULAR COLUMN WITH THE PACKER}

For the exploration and development of oil wells, many wells use packer in the production process. The analysis of the deformation to the strings with the packer is very important for the safety of production.

This paper analyses and calculates the pipe deformation by collecting and analyzing the data obtained from downhole mechanics analysis and calculation.

*Address correspondence to this author at the School of Electrical \& Information Engineering, Northeast Petroleum University, Daqing. Postcard: 163318, China; Tel: 0459-6503160; E-mail: wxfdqpi@163.com

\subsection{Piston Effect}

When the pipe column slides up and down in the packer sealing cavity, tubular column shrinks or stretches because of the piston force generated by pressure difference.

The calculation of the deformation produced by the piston force is as follows:

$\Delta L_{1}=-\frac{\Delta P_{i}\left(A_{3}-A_{2}\right)-\Delta P_{o}\left(A_{3}-A_{1}\right)}{E A_{s}} L$

In equation 1: $A_{3}$ is the cross sectional area of the packer seal cavity; $A_{2}$ is the internal cross sectional area of the tubular column; $A_{1}$ is the external cross sectional area of the tubular column; $A_{s}$ is the cross sectional area of the oil pipe; $E$ is the elastic modulus of steel; $\Delta p_{o}$ is the change of the annular pressure in the upper section of the packer; $\Delta p_{i}$ is the change of the pressure in the packer's upper tubing; $L$ is the total length of tubing.

\subsection{Spiral Bending Effect}

Because of the existence of "virtual force", the downhole tubing often produces spiral bending effect. Spiral bending effect is caused by the pressure in the sealed tube end and the tube wall.

The tubing bending force is $F_{h}$ :

$F_{h}=A_{3}\left(\Delta P_{i}-\Delta P_{o}\right)$

The calculation of the deformation is:

$$
\Delta L_{2}=-\frac{r^{2} F_{h}^{2}}{8 E I W}
$$


In equation 3: $r$ is the difference between the internal radius of the casing pipe and the external radius of the pipe; $W$ is the average mass per unit length of the tubing; $I$ is the moment of inertia of the pipe's cross sectional area on its diameter.

\subsection{Expansion Effect}

The calculation of the deformation caused by expansion effect is as follows:

$\Delta L_{3}=-\frac{\mu}{E} \frac{\Delta \rho_{i}-R^{2} \Delta \rho_{o}-\frac{(1+2 \mu) \sigma}{2 \mu}}{R^{2}-1} L^{2}-\frac{2 \mu}{E} \frac{\Delta P_{i s}-R^{2} \Delta P_{o s}}{R^{2}-1} L$

In equation 4: $\mu$ is the Poisson's ratio of steel, $R$ is the ratio of internal diameter and external diameter; $\Delta \rho_{i}$ is the change of fluid density in the tubing; $\Delta \rho_{o}$ is the change of fluid density in the annular space; $\Delta P_{i s}$ is the change of the oil pressure at the wellhead; $\Delta P_{o s}$ is the change of the casing pressure at the wellhead; $\sigma$ is the pressure drop per length caused by fluxion.

\subsection{Temperature Effect}

When the pressure in the tubular column is greater than the external pressure, the length of the tubular column is changed.

The calculation of the deformation caused by temperature effect is as follows:
$\Delta L_{4}=\beta \Delta T L$

$\Delta T=T_{L}-T_{s}+\frac{1}{2}\left[L \Delta t^{\prime}-(L-9) \Delta t\right]$

In equation 5: $\beta$ is the linear expansion coefficient of steel; $\Delta T$ is the change of temperature in the wellbore; $T_{L}$ is the temperature of the injected fluid at the wellhead; $T_{s}$ is the static temperature at the wellhead; $\Delta t$ is the geothermal gradient; $\Delta t^{\prime}$ is the temperature gradient in the wellbore in the condition of fluid fluxion.

Fig. (1) shows the interface design and experimental results.

\section{THE ANALYSIS OF FRICTIONAL RESISTANCE OF TUBULAR COLUMN}

Downhole drag and torque are two key factors in the design and construction of the well. Analysis of the frictional resistance of tubular column is very important in the design and implementation of oil wells; therefore, it has received wide attention in the upstream industry. Frictional resistance analysis before drilling is one of the starting points of the feasible study of oil well, which is also the basis for the selection and upgrading of the drilling equipment. Frictional resistance analysis after drilling is the basis for the optimization of the casing program [7]. According to the well depth, azimuth, inclination and other borehole data, the system analyzes the frictional resistance of tubular column under the conditions that the working condition is rotary

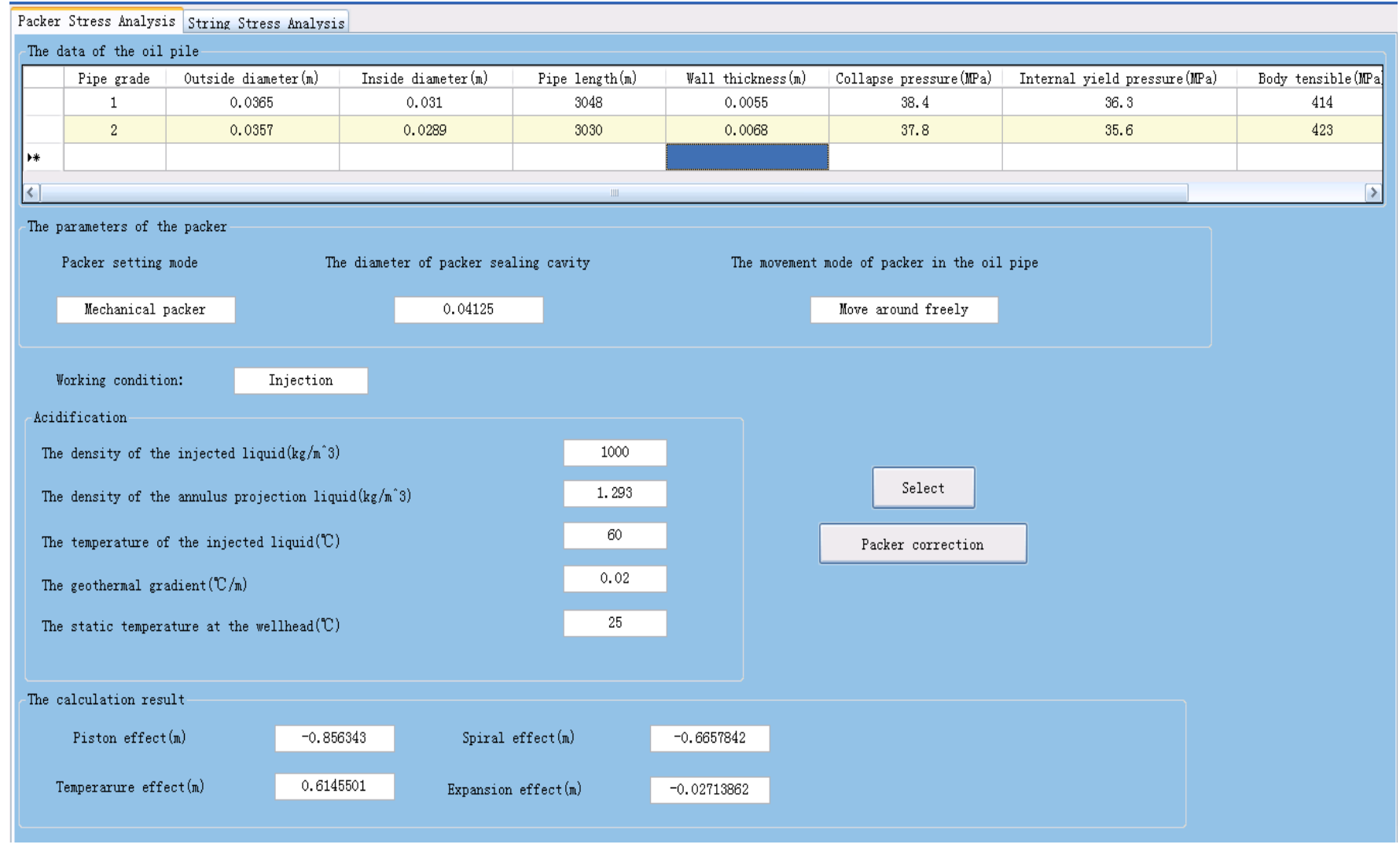

Fig. (1). The analysis of the deformation to the tubular column with the packer. 
drilling, mechanical model is of steel and trajectory curve is natural. The analysis of frictional resistance of tubular column mainly includes two parts: one part consists of static frictional resistance analysis and the other part comprises of dynamic frictional resistance analysis.

\subsection{Static Frictional Resistance Analysis}

Static frictional resistance analysis of the tubular column mainly includes the calculation of the axial force, lateral pressure and torque. In order to ensure the safety of the tubular column, the pressure on the tubular column should be less than the limit tension and limit torque. The axial force, lateral pressure and torque on the tubular column are described by the curves of the pulling force, torque and well depth. At the same time, through checking the strength of the drill string, it draws the safety coefficient of the tubular column to reflect the security of the tubular column. The inclination and the curvature are two important parameters in the analysis of frictional resistance of tubular column. In order to reflect its relationship with frictional resistance, it draws these two parameters in the curve. In order to reflect the bending state of the tubular column, it also draws the stability of the tubular column in the curve. The results of static analysis are mainly used for the design and selection of drill string.

\subsubsection{The Calculation of the Curvature}

Borehole curvature is an important index for measuring the build angle capacity. Understanding of the formation and deflecting characteristics and evaluation of the quality of the well bore constitute the basis for the frictional resistance analysis, and design and monitoring of well path [8]. In the condition of drilling problem, the overall bending degree of the borehole trajectory is often inspected which is the calculation of the average borehole curvature.

The calculation formula of the curvature is as follows:

$K=\sqrt{\left(\frac{\Delta \alpha}{\Delta L}\right)^{2}+\left(\frac{\Delta \varphi}{\Delta L}\right)^{2} \sin ^{2}{ }^{-}}$

In equation 7, $\Delta \alpha=\alpha_{2}-\alpha_{1} ; \Delta \varphi=\varphi_{2}-\varphi_{1} ; \Delta L=L_{2}-L_{1} ; \alpha_{1}$ is the deviation angle in the upper measure point; $\alpha_{2}$ is the deviation angle in the lower measure point; $\varphi_{1}$ is the azimuth in the upper measure point; $\varphi_{2}$ is the azimuth in the lower measure point.

\subsubsection{The Calculation of the Axial Force, Lateral Pressure and Torque}

The tubular column unit in the three-dimensional well bore is shown in Fig. (2).

In Fig. (2), $\mathrm{T}$ is the axial force; $\alpha$ is the deviation angle; $\varphi$ is azimuth; $\theta$ is the total angle change of the tubular column unit; $L_{s}$ is the length of the tubular column unit; $q$ is the effective weight of the tubular column unit; $\tau$ is tangent vector; $n$ is normal vector; $m$ is binormal vector.

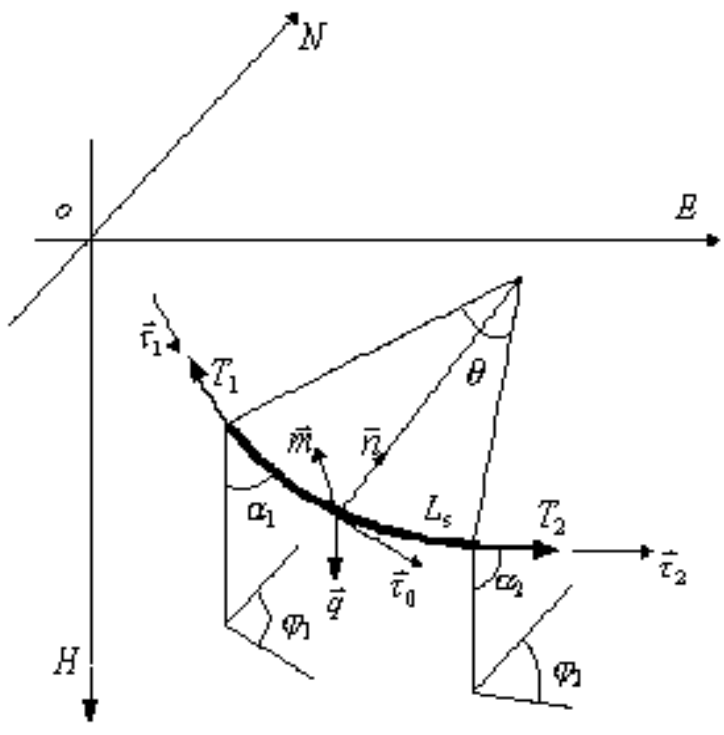

Fig. (2). The tubular column unit in the three-dimensional wellbore.

When the axial force in the lower measure point of the tubular column unit and the lateral force of the tubular column unit are given, the calculation formula of the axial force in the upper measure point is:

$T_{1}=T_{2}+\left[L_{s} / \cos (\theta / 2)\right] \cdot\left[q \cos \alpha \pm \mu\left(F_{E}+F_{n}\right)\right]$

In equation 8: $F_{E}=11.3 E I K^{3} ; L_{s}$ is the length of the tubular column unit; $q$ is the effective weight of the tubular column unit; $\mu$ is the frictional resistance coefficient between wellbore and tubular column;

$\theta=\arccos \left[\cos \alpha_{1} \cos \alpha_{2}+\sin \alpha_{1} \sin \alpha_{2} \cos \left(\varphi_{2}-\varphi_{1}\right)\right]$

is the total angle change of the tubular column unit; $\alpha_{1}$ and $\alpha_{2}$ stand for the deviation angle in the upper measure point and the deviation angle in the lower measure point respectively; $\varphi_{1}$ and $\varphi_{2}$ stand for the azimuth in the upper measure point and the deviation angle in the lower measure point respectively; $F_{E}$ is the lateral force caused by the bending deformation of the wellbore; $K$ is the curvature of the tubular column unit.

The total lateral force in the whole angle plane is:

$F_{n d p}=-\left(T_{1}+T_{2}\right) \sin \frac{\theta}{2}+n_{3} L_{s} q$

In equation 9: $n_{3}$ is the component of the normal vector in the vertical direction.

The total lateral force in the bi-normal direction is:

$F_{n p}=m_{3} q L_{s}$ 
In equation 10: $m_{3}$ is the component of the binormal vector in the vertical direction.

The total lateral force of a tubular column unit in the three-dimensional wellbore is the vector sum of the total lateral force in the whole angle plane and the total lateral force in the bi-normal direction because the two forces are perpendicular to each other. The calculation formula of the total lateral force of a tubular column is:

$$
F_{n}=\frac{\sqrt{F_{n d p}^{2}+F_{n p}^{2}}}{L_{s}}
$$

The calculation formula of the torque in the upper measure point of the tubular column is given by equation 12 :

$$
M_{T 1}=M_{T 2}+\frac{\mu F_{n} L_{s} D_{t j}}{2}
$$

In equation 12: $M_{T 1}$ and $M_{T 2}$ respectively stand for the torque in the upper measure point and the torque in the lower measure point respectively. $D_{t j}$ is the external diameter of the tubular column collar; $\mu$ is the coefficient of the sliding frictional resistance.

\subsubsection{The Calculation of the Safety Factor}

Through the safety factor, the state of the tubular column can be accurately judged. There are many calculation models for the safety factor but some of them are complex. This paper, on the basis of collection and analysis of the previous researches summarizes a set of complete and clear theories to calculate the safety factor.

$n=\frac{\sigma_{s}}{\sigma_{i \max }}$

In equation 13: $n$ is the safety factor; $\sigma_{s}$ is the yield limitation of the material; $\sigma_{\text {imax }}$ is the maximum stress intensity in the cross-section.

$\sigma_{\text {iamx }}=\max \left[\sigma_{x d}\right]$

According to the fourth strength theory, the calculation of the corresponding force in the cross-section is:

$\sigma_{x d}=\frac{1}{\sqrt{2}}\left[\left(\sigma_{F}+\sigma_{m}-\sigma_{r}\right)^{2}+\left(\sigma_{F}+\sigma_{m}-\sigma_{\theta}\right)^{2}+\left(\sigma_{r}-\sigma_{\theta}\right)^{2}\right]^{\frac{1}{2}}$

In equation 15: $\sigma_{F}$ is the axial force in the cross-section of the tubular column; $\sigma_{m}$ is the bending force in the crosssection of the tubular column; $\sigma_{r}$ is the radial force in the cross-section of the tubular column; $\sigma_{\theta}$ is the hoop force in the cross-section of the tubular column.

The calculation of the axial force is:

$\sigma_{F}=\frac{F_{a}}{A_{o i}}$
In equation: $F_{a}$ is the true axial force in the cross-section of the tubular column; $A_{o i}$ is the cross-sectional area of the tubular column.

The calculation of the radial force is:

$\sigma_{r}=\frac{P_{i} r_{i}^{2}-P_{o} r_{o}^{2}}{r_{o}^{2}-r_{i}^{2}}-\frac{r_{o}^{2}}{r_{o}^{2}-r_{i}^{2}}\left(P_{i}-P_{o}\right)$

The calculation of the hoop force is:

$\sigma_{\theta}=\frac{P_{i} r_{i}^{2}-P_{o} r_{o}^{2}}{r_{o}^{2}-r_{i}^{2}}+\frac{r_{o}^{2}}{r_{o}^{2}-r_{i}^{2}}\left(P_{i}-P_{o}\right)$

In equation 17 and equation 18: $P_{i}$ is the internal pressure of the tubular column; $P_{o}$ is the external pressure of the tubular column; $r_{i}$ is the internal diameter of the tubularcolumn; $r_{o}$ is the external diameter of the tubular column.

The calculation of the bending force is:

$\sigma_{m}=\frac{F_{e} \times r \times r_{o}}{2 \times I}$

In equation 19: $F_{e}$ is the effective axial force in the crosssection of the tubular column; $r$ is the difference between the internal radius of the casing pipe and the external radius of the pipe; $r_{o}$ is the external diameter of the tubular column; $I$ is the moment of inertia of the pipe's cross sectional area on its diameter which takes the value as $0.67 \times 10^{-6} \mathrm{~cm}^{4}$.

The calculation of the effective axial force in the equation (19) is:

$F_{e}(z)=F_{a}(z)+P_{i}(z) A_{i}-P_{o}(z) A_{o}$

In equation 20: $P_{i}$ is the pressure of the liquid in the tubular column; $P_{o}$ is the pressure of the liquid outside the tubular column; $A_{i}$ is the internal cross sectional area of the tubular column; $A_{o}$ is the external cross sectional area of the tubular column.

When the internal liquid density, external liquid density, internal liquid flow velocity and external liquid flow velocity are constant, the distributions of the internal and external fluid pressure are:

$$
\begin{aligned}
& P_{i}(z)=\rho_{i} g z-\frac{\rho_{i} \lambda_{i} \mu_{i m}{ }^{2}}{4 r_{i}} z+P_{i o} \\
& P_{o}(z)=\rho_{o} g z-\frac{\rho_{o} \lambda_{o} \mu_{o m}{ }^{2}}{4 r_{o}} z+P_{o o}
\end{aligned}
$$

In equation 21: $P_{i o}$ is the internal pressure of the wellhead; $P_{o o}$ is the external pressure of the wellhead.

The dimensionless frictional resistance of the fluid in the tubular column is: 
$\lambda_{i(o)}= \pm \frac{64}{R_{i(o)}}\left(R_{i(o)} \leq 3 \times 10^{3}\right)$

$\lambda_{i(o)}= \pm 0.3164 R_{i(o)}^{-0.25}\left(R_{i(o)}>3 \times 10^{3}\right)$

The calculation of the Reynolds coefficient is:

$R_{i(o)}=\frac{2 \varphi_{i(o)} r_{i(o)} \mu_{i m(o m)}}{\mu_{i}}$

In equation 23: $\mu_{i}$ is the kinematic viscosity coefficient of the fluid; the significance of the other parameters is the same as above.

\subsubsection{The Calculation of the Stability}

The tubing string in the wellbore often works in a compressed state at some intervals. The precise analysis of the stress and deformation of the tubular column is helpful for optimizing the design [9].

The discriminated formula of the stability of the tubular column is as follows:

When, $F_{\tau}<2 \sqrt{E I \frac{q \sin \alpha}{T_{b}}}$ [24], the tubular column is in a linear steady state, else the tubular column is in a bent state.

In the formula: $F_{\tau}$ is the axial force; $E I$ is the flexural rigidity; $q$ is the line buoyant weight; $\alpha$ is the deviation angle; $T_{b}$ is the one half of the difference between well diameter and tubular column diameter.

The data of the well trajectory are shown in Table 1.

Fig. (3) shows the interface design and experimental results.

\subsection{Dynamic Frictional Resistance Analysis}

Dynamic frictional resistance analyzes the pulling force and the torque at the wellhead. Static frictional resistance analyzes the drag and torque at every point of the drilling string when it drills into the well. Dynamic frictional resistance analysis calculates the wellhead pulling force and wellhead torque at every depth point of the well when the drilling string drills from the wellhead to the bottom. Therefore, by conducting multiple static analysis and calculating the results of the wellhead tension and wellhead torque, the dynamic analysis can be completed. The change of the dynamic load is important for drilling design and is also the basis of the design of the drilling string and of selection of the drilling plan and the equipment.

In this system, the result of the dynamic frictional resistance is described by the curves of the wellhead pulling force, wellhead torque and well depth.

Fig. (4) shows the interface design and experimental results.
Table 1. The data of well trajectory.

\begin{tabular}{|c|c|c|}
\hline Well Depth (m) & Deviation Angle $\left({ }^{\circ}\right)$ & Azimuth $\left(^{\circ}\right)$ \\
\hline 0 & 17.5 & 260 \\
\hline 50 & 18.5 & 262 \\
\hline 100 & 17.5 & 263 \\
\hline 150 & 18 & 268 \\
\hline 200 & 19 & 269 \\
\hline 250 & 21 & 266 \\
\hline 300 & 18 & 255 \\
\hline 350 & 17 & 265 \\
\hline 400 & 18.5 & 263 \\
\hline 450 & 21 & 265 \\
\hline 500 & 18 & 257 \\
\hline 550 & 18.5 & 261 \\
\hline 600 & 18.8 & 263 \\
\hline 650 & 18 & 268 \\
\hline 700 & 19 & 269 \\
\hline 750 & 21 & 266 \\
\hline 800 & 18 & 255 \\
\hline 850 & 17 & 265 \\
\hline 900 & 18.5 & 263 \\
\hline 950 & 17.5 & 257 \\
\hline 1000 & 16 & 263 \\
\hline
\end{tabular}

\section{CONCLUSION}

This system mainly used the modern mechanics theory as the basis model and from two processes analyzed the stress condition of the tubular column. One was the analysis of the deformation to the tubular column with the packer; the other process was the analysis of frictional resistance of tubular column. Through the calculation of the deformation of the tubular column, axial force, torque and lateral force, the stress condition of the tubular column was analyzed. The calculation results of the system can be used to design construction technology, select tubular column and check the security.

This system possesses advantages of fast calculation, simple operation, friendly user interface and accurate calculation. It is robust and has good practicability. It can provide a comprehensive and reliable result for the mechanical analysis of the well. Usage of this system can improve the efficiency and quality of work.

\section{CONFLICT OF INTEREST}

The authors confirm that this article content has no conflict of interest.

\section{ACKNOWLEDGEMENTS}

This work is supported by the National Science \& Technology Support Plan Major Project under Grant No. 2012BAH12B03. 


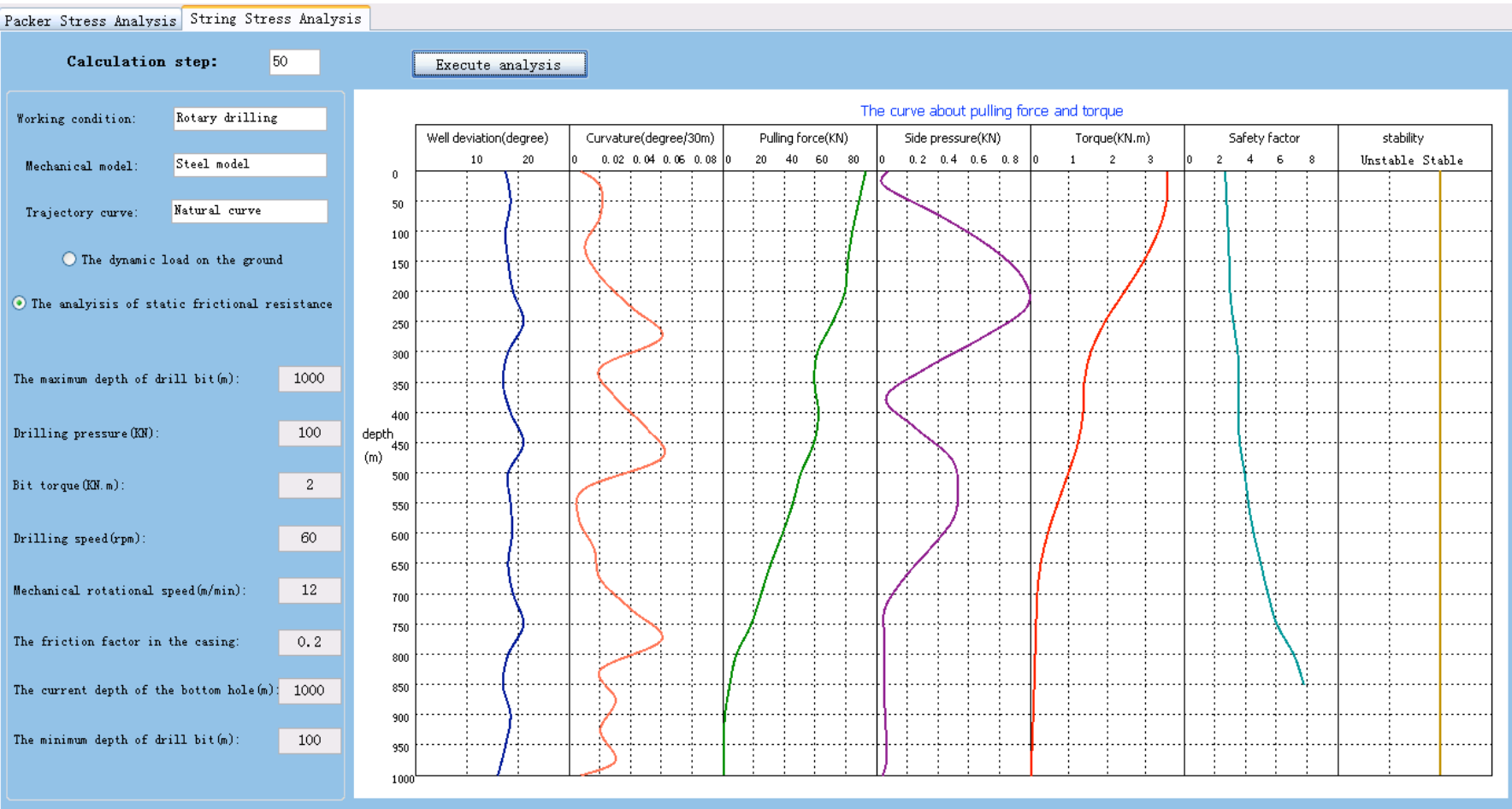

Fig. (3). Static frictional resistance analysis.

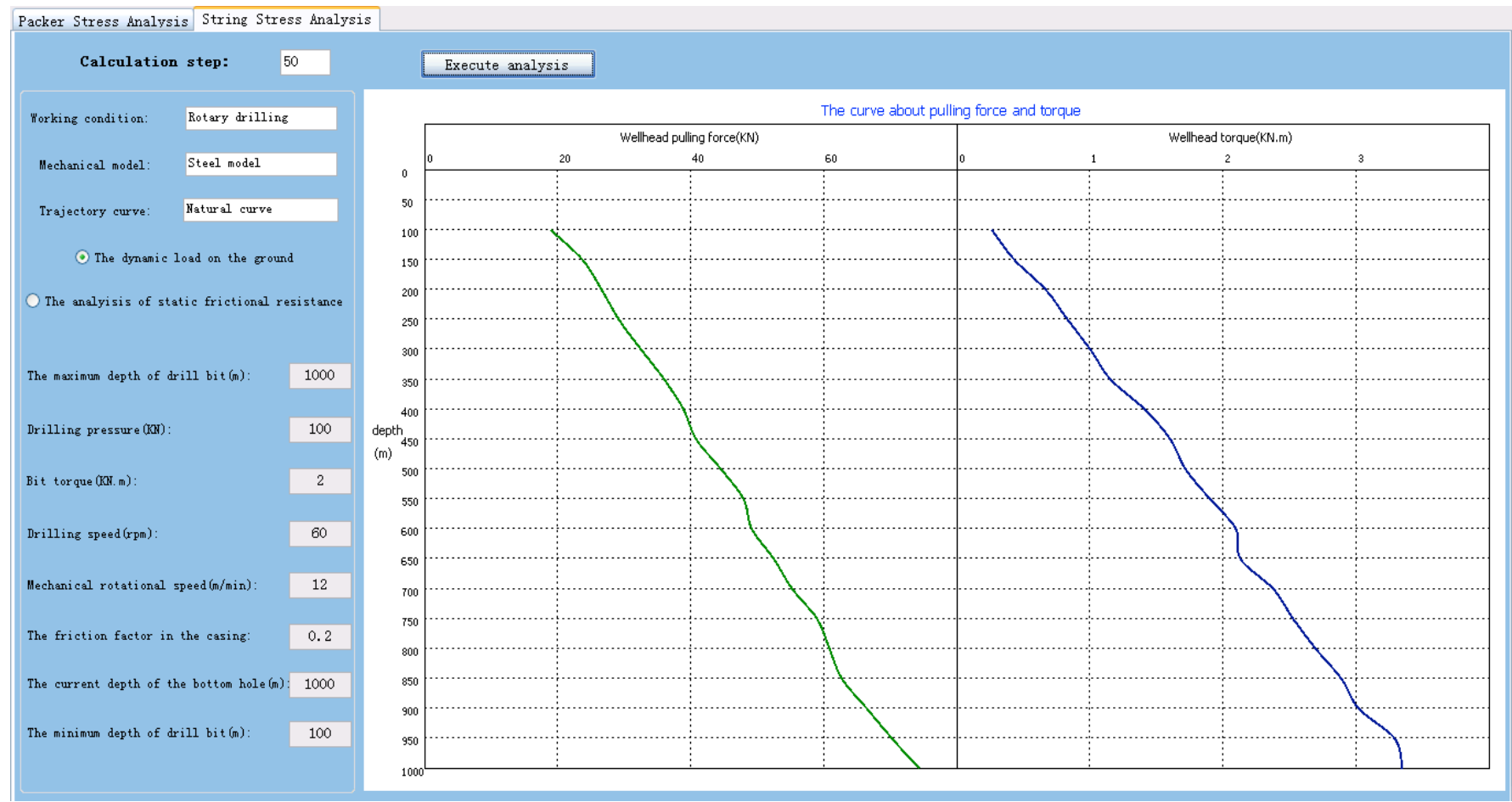

Fig. (4). Dynamic frictional resistance analysis.

\section{REFERENCES}

[1] W. Yong, L. Shuzhi, Z. Chun, G. Lin, Y. Zhengrong and Z. Zhihong, "Stress and Deformation analysis on tubular with packers", Inner Mongulia Petrochemical Industry, vol. 36, pp. 236239, April, 2010.

[2] L. Qi, J. Jianxun, S. Qi, Z. Guoshu and L. Yougang, "Calculation analysis on force deformation of packer string with free movement", Fault-Block Oil \& Gas Field, vol. 14, pp. 61-63, January, 2007.
[3] L. Qindao, X. Guangping and Z. Juan, "Calculation analysis of amount of deformation of free movement packer string", Drilling \& Production Technology, vol. 25, pp. 62-63, January, 2002.

[4] A. Lubinski and W.S. Althouse and J.L. Logan, "Helical buckling of tubing sealed in packers ", Journal of Petroleum Technology, vol. 14, pp. 655-670, April, 2013.

[5] D. J. Hammerlindl, "Packer-to-Tubing forces for intermediate packers”, Journal of Petroleum Technology, vol. 32, pp. 515-527, March, 1980. 
[6] D.J. Hammerlindl, "Movement, Forces, and Stress associated with combination tubing strings sealed in packers", Journal of Petroleum Technology, vol. 29, pp. 195-208, February, 1977.

[7] G. Deli, T. Chengjin, and L. Wenyong, "Research on numerical analysis of drag and torque for Xijiang extended reach wells in South-China Sea ", Oil Drilling \& Production Technology, vol. 25, pp. 7-12, May, 2003.
[8] L. Xiushan, "Average borehole curvature calculation of hole trajectory", Oil Drilling \& Production Technology, vol. 27, pp. 1115, May, 2005

[9] L. Zifeng, "Stability and criss cross curing of pipe pile of oil gas well", West-china Exploration Engineering (Geotechnical Drilling and Digging Mining Engineering), vol. 9, pp. 23-25, March, 1997.

(C) Zhao et al.; Licensee Bentham Open.

This is an open access article licensed under the terms of the Creative Commons Attribution Non-Commercial License (http://creativecommons.org/licenses/ by-nc/4.0/) which permits unrestricted, non-commercial use, distribution and reproduction in any medium, provided the work is properly cited. 